\title{
Potencial da Moringa oleifera Lam (Moringaceae) como fonte de antioxidante natural para biocombustível.
}

\author{
F. R. M. FRANÇA ${ }^{1}$, D. S. MENEZES ${ }^{2}$; J. J. S. MOREIRA ${ }^{3}$, G. F. DA SILVA ${ }^{2}$, S. T. \\ BRANDÃO $^{1}$, \\ ${ }^{1}$ Universidade Federal da Bahia, Departamento de Engenharia Química \\ ${ }^{2}$ Universidade Federal de Sergipe, Departamento de engenharia Química \\ ${ }^{3}$ Universidade Federal de Sergipe, Pós Graduação em Ciência da Propriedade Intelectual
}

\begin{abstract}
RESUMO - Extratos de ervas têm sido estudados devido ao poder antioxidante atribuído ao teor de Compostos fenólicos. A viabilidade do uso de biocombustíveis já foi comprovada tornando-se necessário controlar a sua qualidade. O presente trabalho objetiva determinar o teor de compostos fenólicos, e analisar a decomposição térmica do extrato de Moringa oleifera Lam. Folhas de moringa, secas a 40,70 e $100^{\circ} \mathrm{C}$ foram submetidas à extração a frio em etanol para posterior aplicação como aditivo em biocombustíveis. O conteúdo de compostos fenólicos totais, o perfil de compostos fenólicos e a estabilidade térmica foram determinados. Os extratos obtidos a 40 e $70^{\circ} \mathrm{C}$ mostraram maior potencial com teor de compostos fenólicos de 17,04 e 14,12 ugEqAG/mL respectivamente. Vitexina, Quercitrina, Rutina e Catequina foram identificados. $\mathrm{Na}$ análise térmica o extrato se decompôs a $92,63{ }^{\circ} \mathrm{C}$. As medidas de período de indução mostraram que o extrato aumentou a estabilidade oxidativa do biodiesel. Os resultados indicam o extrato da folha da Moringa oleifera Lam como fonte potencial de antioxidante natural para biocombustível.
\end{abstract}

\section{INTRODUÇÃO}

Os antioxidantes são substâncias que impedem ou minimizam a formação de compostos como peróxidos, aldeídos, cetonas, dímeros e polímeros, que são produtos formados por termo-oxidação de óleos e gorduras e atuam impedindo a etapa inicial da autoxidação, que corresponde à formação de radicais livres, removendo-os do meio (FOOD TECHNOLOGY, 1994; e WAYNER et al, 1985). Algumas substâncias presentes em fontes naturais são capazes de agir como antioxidantes como vitaminas, compostos nitrogenados (alcalóides, aminoácidos, peptídeos) e compostos fenólicos. Dentre os mais importantes, sob o ponto de vista tecnológico, podem ser citados os tocoferóis (vitamina E), os carotenóides, alguns ácidos orgânicos como, ácido cítrico, ácido ascórbico (vitamina C), e os flavonóides (AMAROWICZ et al., 2004; LUZIA e JORGE, 2010).

A Moringa oleifera Lamarck é uma espécie perene, da família Moringaceae, originária do nordeste indiano, amplamente distribuída na Índia, Egito, Filipinas, Ceilão,Tailândia, Malásia, Burma, Paquistão, Singapura, Jamaica e Nigéria (PIO CÔRREA, 1984; DUKE, 1987). 
O teor de compostos fenólicos foi avaliado na semente da Moringa Oleífera Lam e os resultados mostraram através da cromatografia liquida a presença de pelo menos 10 compostos fenólicos (ácido gálico , p -cumárico, ácido ferúlico, ácido cafeico, ácido protocatecuico, ácido cinâmico, catequina, epicatequina, vanilina e quercetina) (SINGH et al., 2013).

O estudo da estabilidade oxidativa do biodiesel é de fundamental importância para o controle de sua qualidade, principalmente no que diz respeito ao armazenamento, que é expresso como o período de tempo requerido para alcançar o ponto em que o grau de oxidação aumenta abruptamente, sendo este tempo chamado de período de indução e é expresso em horas (TAN et al., 2002; VELASCO et al., 2004)..

A Moringa é uma planta de múltiplos usos desde suas folhas até as suas sementes, apresentando diferentes propriedades. As folhas de Moringa são fonte de uma alimentação rica em proteínas tanto para humanos quanto para animais. Além disso, a extração de óleo da semente viabiliza a possibilidade de utilização dessa matéria prima para produção de biodiesel. As sementes de Moringa Oleífera contêm entre 33 e $41 \%$ m m-1 de óleo (RASHID et al., 2008).

Têm sido relatados estudos empregando a Moringa no tratamento de água para uso doméstico e algumas outras aplicações (MANI et al., 2007). Estudos mostrando o potencial do óleo de Moringa extraído de sementes da Índia e Paquistão para produção de biodiesel foram publicados (AZAM et al., 2005; RASHID et al., 2008).

Diante do exposto o presente trabalho objetiva determinar o teor de compostos fenólicos, e analisar a decomposição térmica do extrato de Moringa oleifera Lam, além de verificar a influencia deste extrato em biodiesel comercial de soja no que diz respeito a sua estabilidade a oxidação.

\section{MATERIAIS E MÉTODOS}

\subsection{Coleta e preparo do material vegetal}

Para a obtenção do extrato antioxidante foi utilizada a folha da Moringa oleífera Lam,coletada na Universidade Federal de Sergipe-UFS no período de janeiro a agosto de 2013. As folhas foram beneficiadas retirando-se os talos e posteriormente submetidas à secagem em estufa com circulação forçada de ar nas temperaturas de 40,70 e $100^{\circ} \mathrm{C}$ por 48 horas. Em seguida as folhas já secas foram pulverizadas e armazenadas em vidros hermeticamente fechados e protegidos da luz. 


\subsection{Extração dos compostos fenólicos com etanol (99,5\%) e acetona P.A.}

A folhas secas foram submetidas à extração submergido em dois tipos de solventes a fim de verificar qual deles teria maior afinidade com os compostos fenólicos, responsáveis pela ação antioxidante, presentes na folha da moringa, o qual variou de acordo com a polaridade de cada solvente empregado. Os solventes utilizados foram álcool etílico PA e acetona. A proporção folha pulverizada x solvente foi de $140 \mathrm{~g}$ de folha para $1 \mathrm{~L}$ de álcool, ficando em repouso por 48horas. A etapa seguinte foi à filtração que consistiu na passagem do extrato por um meio filtrante composto por carvão ativado e em seguida foi levado a rotaevaporação para retirada do solvente álcool. A figura 1 apresenta o fluxograma do processo de extração aplicado.

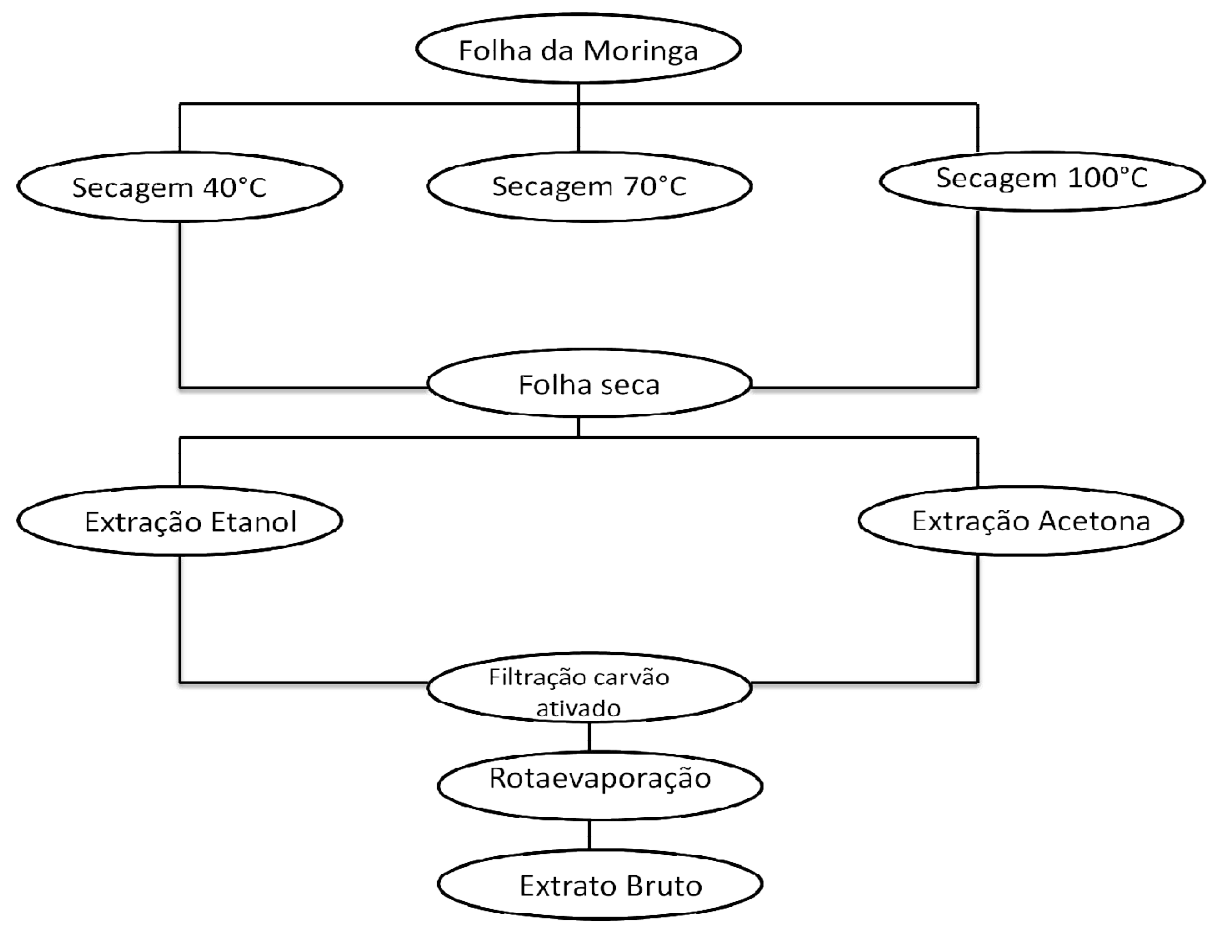

Figura1: Fluxograma do processo de obtenção do extrato da Moringa Oleífera Lam.

\subsection{Caracterização do extrato}

\section{Determinação do teor de fenóis totais}

O teor de compostos fenólicos totais foi determinado de acordo com o método espectrofotométrico de Folin-Ciocauteau descrito por Singleton et al. (1999). Em tubos de ensaio foram adicionados $2,8 \mathrm{~mL}$ de água, $180 \mathrm{ul}$ do reagente Folin- Ciocauteau 0,25N, 150uL 
da amostra (extrato) e $360 \mathrm{uL}$ de carbonato de sódio a 10\%. Após 2 horas ao abrigo da luz, determinações de absorbância a $725 \mathrm{~nm}$ foram realizadas. O teor de fenóis totais das amostras foi determinado pela equação da reta da curva de calibração construída com padrões de ácido gálico $(0,5$ a $10 \mu \mathrm{g} \mathrm{mL}-1)$.

\section{$\underline{\text { Análise Termogravimétrica - TG do Extrato }}$}

O objetivo desta análise foi verificar o perfil da decomposição térmica do extrato desenvolvido, ou seja, o perfil da perda de massa com o aumento da temperatura. As análises de termogravimetria (ATG) do extrato foram conduzidas em um DTG da SHIMADZU modelo $60 \mathrm{H}$. As amostras foram submetidas a aquecimento de 25 a $600^{\circ} \mathrm{C}$ a uma taxa de $10^{\circ} \mathrm{C} / \mathrm{min}$ sob atmosfera inerte de $\mathrm{N}_{2}$ com fluxo de $100 \mathrm{~mL} / \mathrm{min}$. O ensaio utilizou $3,417 \mathrm{mg}$ da amostra em cadinho de platina seguindo metodologia descrita por Santos, 2008.

\section{Determinação da estabilidade à oxidacão}

A estabilidade à oxidação foi medida segundo a norma EN 14112 segundo resolução ANP $\mathrm{n}^{\circ}$. 14/2012, utilizando amostras de $3 \mathrm{~g}$ de biodiesel, as quais foram analisadas sob aquecimento com temperatura de $110{ }^{\circ} \mathrm{C}$ e fluxo de ar constante de $10 \mathrm{~L} \mathrm{~h}-1$. A medida da estabilidade à oxidação foi realizada no equipamento Biodiesel Rancimat 873 da Metrohm e operado com o auxílio do software Biodiesel Rancimat 873.

Biodiesel comercial de soja cedido pela usina Binatural Formosa/Goias foi submetido ao tratamento com o antioxidante natural proveniente da folha da Moringa, obtido a $40^{\circ} \mathrm{C}$, nas concentrações de 100, 500, 1000, 1500 e 2000 ppm e a critério de comparação utilizou-se o antioxidante comercial BHT (butil-hidroxi-tolueno) nas mesmas concentrações.

\section{RESULTADOS E DISCUSSÃO}

Com relação ao rendimento de obtenção dos extratos (Tabela 1) se verifica uma correlação positiva entre a polaridade do solvente e o rendimento da extração. O extrato em etanol $(99,5 \%)$ que é mais polar apresentou maior rendimento em relação à extração com acetona. É importante salientar que o extrato apresenta níveis residuais de clorofila mesmo após filtração em carvão.

\subsection{Teor de compostos Fenólicos}

As concentrações de fenólicos totais, dos três extratos avaliados obtidos em temperaturas diferentes referente a extração com etanol são apresentadas (Tabela 1). De 
acordo com os valores o extrato obtido a $40^{\circ} \mathrm{C}$ foi o que apresentou maior teor de compostos fenólicos, sendo o seu conteúdo $18 \%$ superior ao teor de compostos fenólicos encontrado no extrato obtido a $70^{\circ} \mathrm{C}$ e $80 \%$ superior ao observado no extrato obtido a $100^{\circ} \mathrm{C}$. Esses dados ressaltam que o extrato da Moringa Oleífera Lam obtido a $40^{\circ} \mathrm{C}$ pode atuar como fonte importante de compostos antioxidantes.

Tabela 1: Resultados do teor de compostos fenólicos para os extratos da Moringa Oleífera Lam utilizando solvente etanol PA.

\begin{tabular}{cccc}
\hline $\begin{array}{c}\text { Temperatura de } \\
\text { Obtenção do } \\
\text { Extrato }\left({ }^{\circ} \mathbf{C}\right)\end{array}$ & $\begin{array}{c}\text { Concentração do } \\
\text { Extrato }(\mathbf{g} / \mathbf{m L})\end{array}$ & $\begin{array}{c}\text { Rendimento } \\
(\boldsymbol{\%})\end{array}$ & $\begin{array}{c}\text { Fenólicos Totais } \\
(\mathbf{u g E q A G} / \mathbf{m L})\end{array}$ \\
\hline $\mathbf{4 0}$ & 0,02949 & 4,21 & 17,04 \\
$\mathbf{7 0}$ & 0,02283 & 3,26 & 14,12 \\
$\mathbf{1 0 0}$ & 0,01882 & 3,55 & 2,68 \\
\hline
\end{tabular}

Na tabela 2 encontra-se o teor de compostos fenólicos extraídos com Acetona.

Tabela 2: Resultados do teor de compostos fenólicos para os extratos da Moringa Oleífera Lam utilizando como solvente a acetona.

\begin{tabular}{cccc}
\hline $\begin{array}{c}\text { Temperatura de } \\
\text { Obtenção do } \\
\text { Extrato }\left({ }^{\circ} \mathbf{C}\right)\end{array}$ & $\begin{array}{c}\text { Concentração do } \\
\text { Extrato }(\mathbf{g} / \mathbf{m L})\end{array}$ & $\begin{array}{c}\text { Rendimento } \\
(\boldsymbol{\%})\end{array}$ & $\begin{array}{c}\text { Fenólicos Totais } \\
(\mathbf{u g E q A G} / \mathbf{m L})\end{array}$ \\
\hline $\mathbf{4 0}$ & 0,02782 & 0,19 & 1,44 \\
$\mathbf{7 0}$ & 0,05482 & 0,39 & 9,17 \\
$\mathbf{1 0 0}$ & 0,02704 & 0,19 & 2,75 \\
\hline
\end{tabular}

De acordo com os valores encontrados o extrato obtido a $70^{\circ} \mathrm{C}$ foi o que apresentou maior teor de compostos fenólicos para a acetona, porém em relação à quantidade de fenólicos extraídos pelos dois solventes testados (Tabela 1) e (Tabela 2) verifica-se que o etanol foi mais eficiente que a acetona na extração devido a ser mais polar.

\subsection{Analise Termogravimétrica do extrato}

A análise TG/DTG foi feita com o extrato etanólico obtido a $40^{\circ} \mathrm{C}$ visto que foi o que obteve maior concentração de compostos fenólicos. Foi observado um primeiro evento 
térmico com perda de massa de 7,96\% no intervalo de temperatura de $35,02-96,86{ }^{\circ} \mathrm{C}$, representando o início do processo de decomposição térmica deste material, essa perda inicial pode ser atribuída à perda de umidade. Um segundo evento ocorreu na faixa de temperatura de $96,86-343,91^{\circ} \mathrm{C}$ podendo ser atribuído à volatilização de alguns compostos. Um terceiro evento ocorreu na faixa de temperatura de $343,91-589,48^{\circ} \mathrm{C}$. Esse evento pode ser atribuído à combustão de compostos menos voláteis presentes na amostra (Tabela 3)

Tabela 3: Dados da análise Térmica do extrato etanólico da folha da moringa oleífera Lam.

\begin{tabular}{|c|c|c|c|c|c|c|}
\hline Amostra & $\begin{array}{l}1^{\circ} \text { Perda de } \\
\text { massa }(\%)\end{array}$ & $\begin{array}{l}\text { Faixa } \quad \text { de } \\
\text { Temperatura } \\
\text { da } 1^{\circ} \text { perda } \\
\left({ }^{\circ} \mathrm{C}\right)\end{array}$ & $\begin{array}{l}2^{\circ} \text { Perda } \\
\text { de massa } \\
(\%)\end{array}$ & $\begin{array}{l}\text { Temperatura da } \\
2^{\circ} \text { perda }\left({ }^{\circ} \mathrm{C}\right)\end{array}$ & $\begin{array}{l}3^{\circ} \text { Perda } \\
\text { de massa } \\
(\%)\end{array}$ & $\begin{array}{l}\text { Temperatura } \\
\text { da } 3^{\circ} \text { perda } \\
\left({ }^{\circ} \mathrm{C}\right)\end{array}$ \\
\hline Extrato & 7,96 & 35,02 - 96,86 & 47,26 & $96,86-343,91$ & 34,01 & 343,91- 589,48 \\
\hline
\end{tabular}

O comportamento do antioxidante sintético BHT assim como do Extrato etanólico da Moringa Oleífera Lam pode ser observado na figura 2.
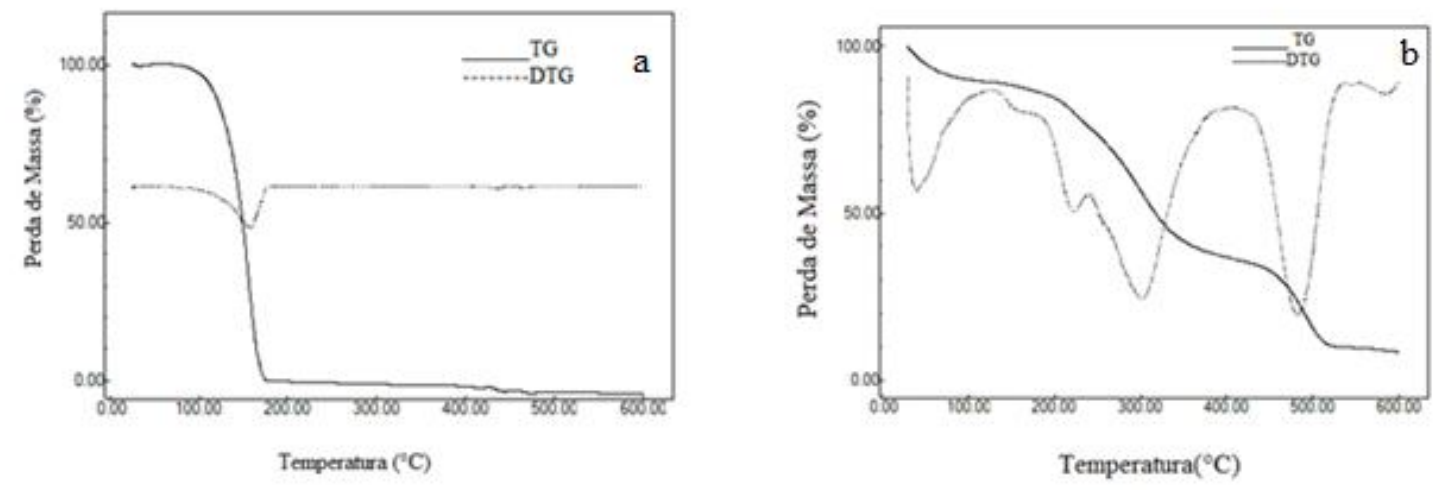

Figura 2 - Curvas TG/DTG para o BHT(a) e para o extrato da folha da moringa(b)

$\mathrm{O}$ antioxidante sintético BHT apresentou o primeiro e único evento em temperatura em torno de $117,91{ }^{\circ} \mathrm{C}$, com a respectiva perda de massa em torno de $97,36 \%$, resultado esperado considerando que o mesmo trata-se de uma substância pura, que praticamente se decompõe em apenas uma faixa de temperatura, sendo mais termo-estável que o extrato vegetal estudado neste trabalho. É possível verificar que extratos são mais instáveis que sustâncias sintéticas, devido à cinética diferenciada dos diversos compostos presentes.

Apesar de a temperatura do extrato apresentar-se inferior à temperatura empregada no ensaio de oxidação acelerada, este fato não impede o uso do extrato como aditivo antioxidante. 


\subsection{Efeito da adição do extrato (antioxidante natural) e do BHT (antioxidante sintético) ao biodiesel comercial de soja}

A Tabela 4 apresenta os valores de Período de indução (PI) obtidos para o biodiesel puro, após adição do aditivo natural (extrato da moringa) e com a adição do BHT.

Tabela 4: Estabilidade oxidativa do biodiesel de Soja (100\%) puro e com adição de diferentes concentrações de extrato e BHT

\begin{tabular}{ccc}
\hline Concentração $(\mathbf{p p m})$ & EXTRATO & BHT \\
\hline $\mathbf{1 0 0}$ & $5,66 \mathrm{~h}$ & $5,73 \mathrm{~h}$ \\
$\mathbf{5 0 0}$ & $5,75 \mathrm{~h}$ & $6,90 \mathrm{~h}$ \\
$\mathbf{1 0 0 0}$ & $5,87 \mathrm{~h}$ & $7,93 \mathrm{~h}$ \\
$\mathbf{1 5 0 0}$ & $5,95 \mathrm{~h}$ & $8,93 \mathrm{~h}$ \\
$\mathbf{2 0 0 0}$ & $6,17 \mathrm{~h}$ & $9,73 \mathrm{~h}$ \\
BIODIESEL PURO & & $4,87 \mathrm{~h}$ \\
\hline
\end{tabular}

O Biodiesel de soja puro (controle) é oxidado após 4,87 horas apresentando valor de PI fora da norma estabelecida ( $<6$ horas). Para todas as concentrações estudadas verificou-se aumento da estabilidade à oxidação (período de indução em horas), porém apenas o de concentração 2000 ppm teve seu valor dentro do limite especificado (6 horas).

A adição do aditivo (extrato da Moringa oleífera Lam) ao biodiesel de soja comercial melhorou a sua estabilidade à oxidação, observando-se também um aumento gradual no período de indução. O limite estabelecido pela Resolução ANP foi atingido na concentração de $2000 \mathrm{ppm}$, ou seja, 0,2\% de antioxidante, obtendo-se um valor de 6,17 h, portanto, foi menos eficiente do que o BHT, mas deve-se considerar que nesse estudo utilizou-se o extrato da moringa bruto, sem nenhum isolamento das substâncias antioxidantes presentes em sua composição.

Apesar do BHT ser mais efetivo do que o extrato da Moringa no aumento da estabilidade à oxidação do biodiesel, este último poderá ser economicamente e ambientalmente interessante como antioxidante alternativo ao BHT e de fonte renovável.

\section{REFERÊNCIAS BIBLIOGRÁFICAS}

AMAROWICZ, R.; PEGG, R. B.; RAHIMI-MOGHADDAM, P.; BARL, B.; WEIL, J. A. Free radical scavenging capacity and antioxidant activity of selected plant species from the Canadian prairies. Food Chemistry. v.84, pág. 551-562, 2004. 
AZAM, M.M.; WARIS, A.; NAHAR, N.M. Prospects and potential of fatty acid methyl esters of some non-traditional seed oils for use as biodiesel in India. Biomass and Bioenergy. v. 29, pág. 293-302, 2005.

DUKE, J. A. Moringaceae: Horseradish-tree, Drumstick-tree, Sohnja, Moringa, Murunga-kai, Mulungay. In: BENGE, M. D. (Ed.) Moringa a Multipurpose Tree that Purifies Water, Boston, Science and Technology for Environment and Natural Resources, pág.19-28, 1987.

FOOD TECHNOLOGY. Current research in natural food antioxidants. INFORM, 5(6), 1994.

LUZIA, D. M. M.; JORGE, N. Potencial antioxidante de extratos de sementes de limão (Citrus limon). Ciênc. Tecnol. Aliment. v. 30, pág. 489 - 493, 2010.

MANI, S.; JAYA, S.; VADIVAMBAL, R. Optimization of solvent extraction of Moringa (Moringa oleifera) seed kernel oil using response surface methodology. Trans Ichem e Part C v. 85, pág. 328- 335, 2007.

PIO CÔRREA, M. Dicionário das Plantas Úteis do Brasil e das Exóticas Cultivadas. Rio de Janeiro: IBDF, v.5, pág. 276-283, 1984.

RASHID, U.; ANWAR, F.; MOSER, B.R.; KNOTHE, G. Moringa oleifera oil: A possible source of biodiesel. Bio. Technol, v. 99, pág 8175-8179, 2008.

GOVARDHAN SINGH R.S., PRADEEP S. NEGI, RADHA C. Phenolic composition, antioxidant and antimicrobial activities of free and bound phenolic extracts of Moringa oleifera seed flour. Journal of functional foods,v.5, pág.1883-1891,2013.

SANTOS, J. R. J. Biodiesel de Babaçu: Avaliação Térmica, Oxidativa e Misturas Binárias. Tese de Doutorado. Universidade Federal da Paraíba. 2008

TAN, C.P.; CHE MAN, Selamat, J; Usoff, M.S.A.; Comparative studies of oxidative stability of edible oils by differential scanning calorimetry and oxidative stability index methods Food Chemistry, v.76, pág. 385-389, 2002.

VELASCO, J.; ANDERSEN, M.L.; SKIBSTED, L.H.; Evaluation of oxidative stability of vegetable oils by monitoring the tendency to radical formation. A comparison of electron spin resonance spectroscopy with the Rancimat method and differential scanning calorimetry, $\mathrm{v}$. 85, pág. 623-632, 2003.

WAYNER, D. D. M.; BURTON, G. W.; INGOLD, K. U.; LOKE, S.J. Quantitative measurement of the total, peroxyl radical - trapping antioxidant capability of human blood plasma by controlled peroxidation, Febs Letters, v.187, pág 33-37, 1985. 